\title{
Survey of various routing approaches based on quality-of-service (QoS) criteria in MANET
}

\author{
Prof. Sunil J. Soni ${ }^{1}$, Dr. J. S. Shah ${ }^{2}$ \\ ${ }^{I}$ (Doctoral Research Scholar, Faculty of Technology, RK University \\ Assistant Professor, Sankalchand Patel College of Engineering, Visnagar) \\ ${ }^{2}$ (Director, Samarth Institute of Technology, Himmatnagar, INDIA)
}

\begin{abstract}
Now a day, Mobile Adhoc Networks (MANET) gained a lot of popularity in the research community. People realized that there is a commercial value in MANETs with the successful solutions of the most basic but important problems in network layer. Mostly all the applications that attracted interest in traditional wired networks (e.g. Audio/Video Transfer, Video Conferencing, Watching Online Movies/Sports, Camera-enabled Messenger, Online Gaming etc.) would attract interest for MANETs as well. Since real-time multimedia communications will be common in MANETs, there has been an increasing motivation on the introduction of QoS in such networks. But, many characteristics of MANETs make QoS provisioning a difficult problem. In MANETs, providing QoS guarantees is very difficult and challenging than in traditional wired networks, mainly due to multihop communications, contention for channel access, node mobility and a lack of central coordination. However, in the last few years, much research attention has focused on providing QoS guarantees in MANET routing protocols. QoS routing protocol/mechanism is key issue in provision of QoS in MANETs. There are number of QoS routing approaches have been proposed in literature, focusing on different QoS metrics but no particular protocol/mechanism provides overall solution. There are some major open research issues in this area such as QoS metric selection and cost function design, Multi-class traffic, Scheduling mechanism at source, Packet prioritization for control messages, QoS routing that allows preemption, and QoS in Heterogeneous networks. There are still many open questions that must be solved to improve the performance of QoS-aware routing protocols such as bandwidth/delay estimation, route discovery, resource reservation, route maintenance, cross-layer design, energy consumption, multicast QoS routing etc.
\end{abstract}

Keywords: Cross-layer Design, Quality-of-Service, MANETs, routing, QoS routing, QoS framework, multicast $Q o S$

\section{Introduction}

Routing in MANET is one of the core problems for data exchange between nodes in networks. In recent years, both the areas of providing quality-of-service and routing in mobile ad-hoc networks have massively increased in importance. Many routing protocols for wireless networks, e.g. AODV or DSR, use besteffort routing, where all nodes within range compete for the shared medium. No guarantees or predictions can be given here on when a node is allowed to send. For quality-of-service (QoS) routing, it is not sufficient to only find a route from a source to one or multiple destinations. This route also has to satisfy one or more QoS constraints. To guarantee these constraints after a route was found, resource reservations on the participating nodes are made.

When MANET development started, QoS provision did not attract much attention and thereby most routing protocols operated on a best effort model. However, with the growing popularity of time-sensitive applications, QoS support becomes much more important than it was, leading to a shift of research interest from best effort routing to QoS provision routing. However, providing QoS guarantees in MANETs is quite challenging due to the dynamic topology, limited bandwidth and energy constraint.

Due to the significant difference in MANETs, the mechanisms for wired networks cannot be mapped to MANETs directly. QoS provision in MANETs is quite challenging and it involves actions in different layers within which the network layer plays a crucial role. The routing protocol in the network layer not only has to find a path, if any, that can satisfy QoS requirements at the beginning of a session but also needs to react to mobility induced route breakages.

Efforts have been made to find a best metric for QoS routing using cross-layer designs but still good generally accepted solution is not achieved. So there is a very good scope of doing research in this area for find a best metric using cross-layer designs, multi-constraints routing metric, multicast QoS routing etc.

\section{QoS In Manet}

Mobile Adhoc Networks (MANETs) has gained a lot of attention in the research community in recent years. Most applications that attract interest for use in current wired networks would attract interest for 
MANETs as well. However, MANETs present unique advanced challenges, including the design of protocols for mobility management, effective routing, data transport, security, power management, and Quality-of-Service (QoS) provisioning. Once these problems are solved, the practical use of MANETs will be realizable. So out of these all issues, authors would like to focus on providing QoS in MANETs and specifically on enhancing routing protocols/mechanisms to support QoS in MANETs.

Since real-time multimedia communications will be common in MANETs, there has been an increasing motivation on the introduction of QoS in such networks. However, many characteristics of MANETs make QoS provisioning a difficult problem. In MANETs, providing QoS guarantees is very difficult and challenging than in traditional wired networks, mainly due to multihop communications, contention for channel access, node mobility and a lack of central coordination. However, in the last few years, much research attention has focused on providing QoS guarantees in MANET routing protocols.

\section{Survey Of Related Work}

In [2], at the signaling level with resource reservation mechanisms independent of the routing protocol. The QoS at the signaling layer is responsible for the coordination of the other QoS layers (MAC and routing) as well as other components such as scheduling or admission control. Signaling level is very essential component for providing QoS in routing protocol.

In [3], the MAC layer is responsible for scheduling and allocating the wireless channel, which eventually will determine the available bandwidth and the packet delay. The bandwidth and packet delay will then affect the decision at the routing layer for link/path selection. The routing layer chooses proper wireless links to relay packets to the destination. The routing decision will change the contention level at the MAC layer. Thus, for a cross-layer design that satisfactorily enhances the network performance, it is essential to highlight the interactions among these layers.

In [4], authors have proposed a novel cross-layer framework for QoS support in WMSNs, which optimizes the functionalities of communication protocols to maximize the number of video stream requests to be delivered while the imposed distortion constraint on the streams are met. QoS requirements are mapped on joint operations of application, network, MAC and link layers of the underlying communication framework.

In [5], it is presented that a cross-layer design that tries to combine the functionality of the routing layer with Medium Access Control (MAC) information and physical layer parameters to provide the routing algorithm with the more accurate information about the environment. The cross-layer design of the proposed routing algorithm in conjunction with an effective resource allocation in the MAC layer operates as a distributed admission control which is able to improve the global performance in the network.

In [6], authors have proposed a unified framework that exploits the physical channel properties and multi-user diversity gain of WMNs and by performing intelligent route selection and connection admission control (CAC) which provides both QoS and GoS (grade-of-service) to a variety of underlying applications. The main difficulty of opportunistic scheduling scheme which is proposed in the paper is while it exploits the wireless channel fluctuations and multiuser diversity gain; it cannot guarantee hard resource reservation required by the network layer to provide QoS. Moreover, routing and scheduling decisions have inherently different operation time scales and this makes difficult the cross layer interaction if QoS needs to be guaranteed.

In [7], cross-layer designs allow information sharing among all of the five layers in order to improve the wireless network functionality, including security, QoS, and mobility. Here, authors have classified crosslayer designs by two ways. On the one hand, by how to share information among the five layers, cross-layer designs can be classified into two categories: non-manager method and manager method. On the other hand, by the organization of the network, cross-layer designs can be classified into two categories: centralized method and distributed method.

In [8], authors have proposed ICMP messages scheme in which desired information is abstracted to parameters, measured by corresponding layers wherever convenient. A new ICMP message is generated only when a parameter changed beyond the thresholds. Since cross-layer communications are carried out through selected "holes" not a general "pipe" as in packet headers scheme, this method seems more flexible and efficient.

In [9], authors have presented the QoS provisioning mechanisms being developed for MANETs. QoS provisioning in MANETs is a Multi-Layer problem. However, most of the protocols for QoS provisioning that are being developed are single layer approaches. This does not provide a comprehensive solution. One of the areas of future research would be to develop the coordination and communication among the various network layers to achieve QoS in MANETs.

In [10], proposed INSIGNIA QOS framework allows packet audio, video and real-time data applications to specify their maximum and minimum bandwidth needs and plays a central role in resource allocation, restoration control, and session adaptation between communicating mobile hosts. 
In [11], authors have discussed the problem of QoS provisioning from the perceptive of three layers application layer, network layer and medium access control (MAC) layer. Main goal was to design a QoS framework which maps QoS parameters across these layers of the protocol stack, to support a standard notion of per-class service guarantees in terms of throughput and delay in ad hoc networks. Further to cope with network dynamics, MAC layer and network layer are made to adapt their behavior based on both dynamic conditions and QoS requirements of admitted flows.

\section{Conclusion}

The rapid growth of video in mobile traffic has resulted in a shift of research interests from best effort service to the provision of higher and better quality of service in MANETs. QoS routing algorithm design is challenging because it has to deal with unfavourable conditions such as time-dependent wireless links, dynamic topology and energy constraints. Considerable efforts have been devoted to this which leads to the emergence of a number of QoS routing techniques.

Generally speaking, two schemes, new protocol design and QoS-aware extension, are adopted to implement QoS routing. New protocol design refers to developing an algorithm with a new methodology while QoS-aware extension means combining QoS guarantee schemes with some well-studied protocols (e.g., DSDV, DSR and AODV).

Real-time applications are generally requires QoS support. Multimedia applications e.g. audio, video etc. has to optimize its performance according to the network conditions. However, some minimum bandwidth should be guaranteed which is used to deliver the basic layer data for multimedia packets. Thus, for QoS support design, admission control, scheduling of multimedia packets and adaptive feedback service are required.

Overall, QoS-aware routing should have the following features that traditional routing does not support:

- obtain resource information from lower layers;

- offer bandwidth information to applications;

- incorporate resource reservation schemes; and

- predict route breaks.

So in this paper, authors have focused on most commonly used routing protocols approaches based on QoS criteria and tried to help researchers to decide and choose best suited routing protocol approaches based on QoS in MANET.

\section{References}

[1] Subir KS, TG Basavaraju, C Puttamadappa. “Adhoc Mobile Wireless Networks, Principles, Protocols and Applications” Auerbach Publications, 2008

[2] S. Lohier, Sidi MS, Yacine GD, et al. "QoS routing in adhoc networks", 2003

[3] Quin Zhang, Ya-qin Zhang. "Cross-Layer Design for QoS Support in Multihop Wireless Networks", Vol.96, No.1, Jan 2008, IEEE Proceedings.

[4] Ghalib A. Shah, Weifa Liang, et al. "Cross-Layer Design for QoS Support in Wireless Multimedia Sensor Networks",Globecom, 2010, IEEE Proceedings.

[5] Maria Canales, Jose Ramon Gallego, et al. "Cross-layer proposal for QoS routing in Mobile Ad-hoc Networks", WPMC, Denmark, 2005.

[6] Chi Harold, Gkelias, et al. "Cross-Layer Design for QoS in Wireless Mesh Networks”, Springer Proceedings, 2009.

[7] Bo Fu, Yang Xiao, et al. "A Survey of Cross-Layer Designs in Wireless Networks", IEEE Communications Surveys \& Tutorials, IEEE, 2013

[8] Qi Wang, Abu-Rgheff. “Cross-Layer Signalling for Next-Generation Wireless Systems”, IEEE Wireless Commun. \& Networking Conference, vol. 2, no. 16C20, March 2003, pp. 1084-1089

[9] S. Marwaha, J. Indulska, et al. "Challenges and Recent Advances in QoS Provisioning, Signaling, Routing and MAC protocols for MANETs", 2010

[10] S. Lee, G. Ahn, "INSIGNIA: An IP-Based Quality of Service Framework for Mobile ad Hoc Networks", JPDC, 2000

[11] N. Sharma, S. Nandi, "A Cross-layer QoS Mapping Framework for Mobile Adhoc Networks", IETE Technical Review, Vol. 25, Issue.6, Dec-2008

[12] R. Goyal, M. Mishra, "Quality of Service Provisioning In MANET Using A Cross-Layer Approach For Routing”, IJCNC, Vol.3, No.3, May 2011

[13] Lei Chen, "Protocols for Supporting Quality of Service in Mobile AdHoc Networks", PhD Thesis, Rochester, New York, 2006

[14] Soni, S.J.; Nayak, S.D., "Enhancing security features \& performance of AODV protocol under attack for MANET," Intelligent Systems and Signal Processing (ISSP), 2013 International Conference on, vol., no., pp.325,328, 1-2 March 2013 doi: 10.1109/ISSP.2013.6526928

[15] Soni, S.J.; Nayak, S.D., "Securing AODV for MANETs using Message Digest with Secret Key (Extended Work)," International Conference on Intelligent Systems \& Data Processing (ICISD), Jan 2011, GCET, VVNagar, Gujarat, INDIA

[16] Soni, S.J.; Swadas, P.B., "Securing AODV for MANETs using Message Digest with Secret Key," International Conference on Computer Networks and Security (ICCNS), 2008, VIT, Pune, INDIA 